1146

\section{USE OF EXHALED CO2 DETECTOR FOR NEONATAL INTUBATION}

A. Gupta, K. Ganesan, S. Oddie

Department of Neonatology, Bradford Royal Infirmary, Bradford, UK

Background: Endotracheal intubation is a common procedure performed in NICU. Sometimes, it is difficult to confirm the successful intubation using standard methods like auscultation. Exhaled CO2 detectors have been widely used in paediatric and adult population and sparingly in neonates. It has been felt that it is an effective confirmation technique for successful intubations.

Aim: To determine whether use of exhaled $\mathrm{CO} 2$ detector during neonatal intubation reduces number of attempts during neonatal intubation and increases the certainty about successful intubation.

Method: Data were collected for each intubation on a pre-designed proforma at tertiary neonatal unit for three months before (Group A) and after introduction of exhaled CO2 detector (Group B). Data were obtained as part of an audit so ethical approval was not required.

Results: Thirty seven intubations were performed in Group A (8 excluded-data inadequate) and twenty five in Group B. There were no statistically significant differences between two groups as far as numbers of attempts are concerned. Certainty of successful intubation increased significantly with the use of exhaled CO2 detector, Group A 22/29 versus Group B 24/25, p value 0.02. We also noted that haemodynamic parameters were similar in both the groups.

Conclusion: Using an exhaled $\mathrm{CO} 2$ detector reduces uncertainty about endotracheal tube placement but does not reduces number of attempts at intubation.

\section{7}

\section{USE OF CRANIAL ULTRASOUND SCAN (CUSS)} IN NEONATAL ABSTINENCE SYNDROME

\author{
A. Silwal, S. Seal \\ Department of Neonatology, Bradford Royal \\ Infirmary, Bradford, UK
}

Background: Anecdotal reports have shown neuroanatomical abnormalities in babies linked to maternal cocaine use. Hence, many neonatal units offer cranial ultrasound scan to babies born to mothers who have used cocaine. There is no strong evidence base for this clinical practice.

Aim: To assess usefulness of cranial ultrasound scans in management of babies born to mothers using cocaine.

Method: Retrospective review of cranial ultrasound findings of neonates born to cocaine using mothers.

Result: Out of fifty six babies born to mothers using cocaine, cranial ultrasound scans were performed on forty four babies. Forty one of these were reported as normal. Echogenic lesions were noted on ultrasound scans in three babies and in two of them, the lesions disappeared/improved. Only one baby was followed up in clinic for three months and was subsequently discharged.

Conclusion: CUSS in neonates whose mothers have used cocaine does not provide any important information regarding neuro-developmental outcome.

1148

\section{EARLY COMUNICATIVE AND LANGUAGE DEVELOPMENT IN PRETERM INFANTS WITHOUT NEUROLOGICAL DAMAGE}

\author{
M. Capobianco, G. Riccio, A. Devescovi, \\ E. Baumgartner
}

Dipartimento di Psicologia, Sapienza, Roma, Italy

Aims: This study aims to explore the role of biological and enviromental factors as predictors of the early comunicative and language development, comparing 12 Italian preterm infants without central nervous system insults and 12 Italian full-term infants.

Method: All participants were assessed longitudinally through the questionnaire "II Primo Vocabolario del Bambino" (PVB), administered monthly to the mothers between 11 and 18 months of age. Assessing preterm infants both chronological age and age correction for prematurity were applied. The measures derived by PVB were: gestures production, words comprehension and production in each age.

Results: Results - on the basis of chronological age - indicated that preterm infants exhibited an 